\title{
A GENERALIZED CONVOLUTION FOR FINITE FOURIER TRANSFORMATIONS ${ }^{1}$
}

A. W. JACOBSON

1. Introduction. The finite sine transformation and the finite cosine transformation of $F(x)$ with respect to $x$ are defined as follows:

$$
\begin{array}{ll}
S\{F(x)\}=\int_{0}^{\pi} F(x) \sin n x d x=f_{s}(n) & (n=1,2, \cdots), \\
C\{F(x)\}=\int_{0}^{\pi} F(x) \cos n x d x=f_{c}(n) & (n=0,1,2, \cdots)
\end{array}
$$

respectively. For example, the sine transforms of the first and second derivatives of $F(x)$ are $S\left\{F^{\prime}(x)\right\}=-n C\{F(x)\}$ and $S\left\{F^{\prime \prime}(x)\right\}$ $=-n^{2} S\{F(x)\}+n\left[F(0)-(-1)^{n} F(\pi)\right]$.

If $F(x)$ in $(-2 \pi, 2 \pi)$ and $G(x)$ in $(-\pi, \pi)$ are bounded and integrable, then the function

$$
F(x) * G(x)=\int_{-\pi}^{\pi} F(x-y) G(y) d y
$$

is called the convolution of $F$ and $G$ on the interval $(-\pi, \pi)$.

If $F(x)$ and $G(x)$ are bounded and integrable on the interval $0 \leqq x \leqq \pi$, and if $F_{1}(x)$ is an odd periodic extension of $F(x)$ and $G_{1}(x)$ an odd extension of $G(x)$, then the product of the sine transforms of $F(x)$ and $G(x)$ can be written in terms of the transform of the convolution as follows:

$$
S\left\{F_{1}(x)\right\} S\left\{G_{1}(x)\right\}=-2^{-1} C\left\{F_{1}(x) * G_{1}(x)\right\} .
$$

See $[1, \text { p. 274 }]^{2}$ and $[2$, p. 270].

2. The Fourier transformation of a generalized convolution. The purpose of this paper is to generalize the above results and to illustrate the use of the generalized convolution. This generalization consists, primarily, of extending the concept of the convolution to any

Presented to the Society, April 16, 1948; received by the editors June 25, 1948.

1 The author wishes to thank Professor R. V. Churchill for his advice in the preparation of this paper. The content of this paper is part of a dissertation submitted in partial fulfillment of the requirements for the degree of doctor of philosophy in the University of Michigan.

2 The numbers in brackets refer to the bibliography. 
integrable function of two variables. The aim here is to obtain a generalization similar to the one made by Bartels and Churchill [3] regarding the Laplace transformation. It will be seen in the next section that with the aid of this generalized convolution, very general steady state boundary value problems can be resolved into simpler problems.

Let $F(x, y)$ be a bounded and integrable function of $x$ and $y$ over the square $0 \leqq x \leqq \pi, 0 \leqq y \leqq \pi$. A generalized Fourier convolution $F^{*}(x)$ of $F(x, y)$ corresponding to the iterated finite sine transformation

$$
\begin{aligned}
S\{S\{F(x, y)\}\} & =\int_{0}^{\pi} \int_{0}^{\pi} F(x, y) \sin n x \sin n^{\prime} y d x d y \\
& =\bar{f}\left(n, n^{\prime}\right) \quad\left(n, n^{\prime}=1,2, \cdots\right)
\end{aligned}
$$

is defined by

$$
F^{*}(x)=-\int_{-\pi}^{\pi} F_{1}(x-y, y) d y,
$$

where $F_{1}(x, y)$ is an odd periodic extension of $F(x, y)$ with respect to $x$ and an odd extension with respect to $y$.

In case $F(x, y)=F_{1}(x), G_{1}(y)$, the function $F^{*}(x)$ is the convolution (1) of $F_{1}$ and $G_{1}$.

Theorem. If $F(x, y)$ is a bounded and integrable function of $x$ and $y$ over the square $0 \leqq x \leqq \pi, 0 \leqq y \leqq \pi$ and if $n^{\prime}=n$, then

$$
S\{S\{F(x, y)\}\}=2^{-1} C\left\{F^{*}(x)\right\} .
$$

Proof. Over the square $0 \leqq \lambda \leqq \pi, 0 \leqq \mu \leqq \pi$ the iterated integral (3) can be written as

$$
\begin{aligned}
2^{-1} \int_{0}^{\pi} \int_{0}^{\pi} F(\lambda, \mu) \cos & n(\lambda-\mu) d \lambda d \mu \\
& -2^{-1} \int_{0}^{\pi} \int_{0}^{\pi} F(\lambda, \mu) \cos n(\lambda+\mu) d \lambda d \mu .
\end{aligned}
$$

In the first integral set $\lambda-\mu=x$ and $\mu=y$. Then the lines $\lambda=0$ and $\lambda=\pi$ in the $\lambda \mu$-plane become the lines $x+y=0$ and $x+y=\pi$ in the $x y$-plane. Let $A$ denote the parallelogram bounded by the last two lines and the lines $\mu=0, \mu=\pi$. Since the Jacobian for this change of variables is unity, the first of the integrals (5) becomes

$$
\iint_{\boldsymbol{A}} F(x+y, y) \cos n x d x d y,
$$


or

$$
\begin{aligned}
& \int_{-\pi}^{0} \cos n x d x \int_{-x}^{\pi} F(x+y, y) d y \\
& +\int_{0}^{\pi} \cos n x d x \int_{0}^{\pi-x} F(x+y, y) d y .
\end{aligned}
$$

If in the first integral in (6) $x$ is replaced by $-x$, then the first integral in (5) can be written

$$
2^{-1} \int_{0}^{\pi} \cos n x d x\left[\int_{0}^{\pi-x} F(x+y, y) d y+\int_{x}^{\pi} F(y-x, y) d y\right] .
$$

By setting $\lambda+\mu=x$ and $\mu=y$ in the second integral in (5), it can be shown to take the form

$$
2^{-1} \int_{0}^{\pi} \cos n x d x\left[\int_{0}^{x} F(x-y, y) d y+\int_{\pi-x}^{\pi} F(2 \pi-x-y, y) d y\right] .
$$

Hence for the iterated sine transformation (3) there results the equation

$$
\begin{aligned}
S\{S\{F(x, y)\}\}= & 2^{-1} \int_{0}^{\pi} \cos n x d x\left[-\int_{0}^{x} F(x-y, y) d y\right. \\
& +\int_{x}^{\pi} F(y-x, y) d y+\int_{0}^{\pi-x} F(x+y, y) d y \\
& \left.-\int_{\pi-x}^{\pi} F(2 \pi-x-y, y) d y\right] .
\end{aligned}
$$

If $F_{1}(x, y)$ is an odd periodic extension of $F(x, y)$ with respect to $x$ with period $2 \pi$ and an odd extension with respect to $y$, then the quantity in brackets reduces to

$$
-\int_{-\pi}^{\pi} F_{1}(x-y, y) d y
$$

which according to definition (4) is the generalized convolution $F^{*}(x)$ of $F(x, y)$. So

$$
S\{S\{F(x, y)\}\}=2^{-1} C\left\{F^{*}(x)\right\} .
$$

If, in (7), $I_{1}, I_{2}, I_{3}$, and $I_{4}$ denote the four integrals in brackets, it can be shown under the same conditions as in the above theorem that

$$
S\{C\{F(x, y)\}\}=2^{-1} S\left\{F^{*}(x)\right\},
$$


where $F^{*}(x)=I_{1}-I_{2}-I_{3}+I_{4} ;$ and $C\{C\{F(x, y)\}\}=2^{-1} C\left\{F^{*}(x)\right\}$, where $F^{*}(x)=I_{1}+I_{2}+I_{3}+I_{4}$; and finally

$$
C\{S\{F(x, y)\}\}=2^{-1} S\left\{F^{*}(x)\right\},
$$

where

$$
F^{*}(x)=I_{1}+I_{2}-I_{3}-I_{4}
$$

If $F(x, y)$ is also periodic with respect to $y$ with period $2 \pi$, then

$$
S\{C\{F(x, y)\}\}=C\{S\{F(x, y)\}\} .
$$

3. Resolution of steady state temperature problem. With the aid of the generalized convolution a very general steady temperature problem can be resolved into a problem with simpler boundary conditions and source function. Furthermore, this method makes it possible to extend the Duhamel integral formula from time to space coordinates.

Consider the function $V\left(x, y_{1}, y_{2}\right)$. Let $\Lambda$ and $\lambda$ denote the differential operators defined by

$$
\Lambda\{V\}=C_{0} V+\sum_{i=1}^{2} C_{i} \frac{\partial}{\partial y_{i}}\left(K_{i} \frac{\partial V}{\partial y_{i}}\right)
$$

and

$$
\lambda\{V\}=c_{0} V+\sum_{i=1}^{2} c_{i} \frac{\partial V}{\partial y_{i}},
$$

where the coefficients of $V$ and its derivatives are functions of $y_{1}$ and $y_{2}$ only. Let the region $R$ be bounded by a cylinder whose elements are parallel to the $x$-axis and whose bases are $x=0$ and $x=\pi$. Let $Q$ denote an arbitrary point on the lateral surface, and $P_{0}$ and $P_{1}$ arbitrary points on the bases. If the coefficients of the second partial derivatives of $V$ with respect to $y_{1}$ and $y_{2}$ are positive, then the following boundary value problem represents a very general steady state temperature problem:

$$
\begin{aligned}
& \frac{\partial^{2} V}{\partial x^{2}}\left(x, y_{1}, y_{2}\right)+\Lambda\{V\}=F\left(x, y_{1}, y_{2}\right), \quad x>0, \\
& \lambda\{V(x, Q)\}=G(x, Q), \quad x>0, \\
& V\left(+0, y_{1}, y_{2}\right)=H_{0}\left(P_{0}\right), \quad V\left(\pi-0, y_{1}, y_{2}\right)=H_{1}\left(P_{1}\right),
\end{aligned}
$$

where $F, G, H_{0}, H_{1}$ are known functions. In the second of these equa- 
tions it is understood that $\lambda\{V(x, Q)\}$ represents the limit of $\lambda\{V(x, P)\}$ as the interior point $P$ approaches the point $Q$ on the lateral surface in a prescribed manner.

Let the corresponding small letter denote the sine transform of the function with respect to $x$. Applying the sine transformation to problem (A) yields:

$$
\begin{aligned}
-n^{2} v\left(n, y_{1}, y_{2}\right)+ & n\left[H_{0}\left(P_{0}\right)-(-1)^{n} H_{1}\left(P_{1}\right)\right] \\
& +\Lambda\left\{v\left(n, y_{1}, y_{2}\right)\right\}=f\left(n, y_{1}, y_{2}\right), \\
\lambda\{v(n, Q)\}= & g(n, Q) .
\end{aligned}
$$

Let the function $U\left(x, x^{\prime}, y_{1}, y_{2}\right)$, depending on the parameter $x^{\prime}$ independent of $x, y_{1}, y_{2}$, be the solution in the region $R$ of the following problem:

$$
\begin{gathered}
\frac{\partial^{2} U}{\partial x^{2}}\left(x, x^{\prime}, y_{1} y_{2}\right)+\Lambda\{U\}=\frac{\pi-x}{\pi} F\left(x^{\prime}, y_{1}, y_{2}\right), \\
\lambda\left\{U\left(x, x^{\prime}, Q\right)\right\}=\frac{\pi-x}{\pi} G\left(x^{\prime}, Q\right), \\
U\left(+0, x^{\prime}, y_{1}, y_{2}\right)=\frac{\pi-x^{\prime}}{\pi} H_{0}\left(P_{0}\right), \\
U\left(\pi-0, x^{\prime}, y_{1}, y_{2}\right)=\frac{\pi-x^{\prime}}{\pi} H_{1}\left(P_{1}\right) .
\end{gathered}
$$

The sine transformation of problem (B) with respect to $x$ gives:

$$
\begin{gathered}
-n^{2} u\left(n, x^{\prime}, y_{1}, y_{2}\right)+n \frac{\pi-x^{\prime}}{\pi}\left[H_{0}\left(P_{0}\right)-(-1)^{n} H_{1}\left(P_{1}\right)\right] \\
+\Lambda\left\{u\left(n, x^{\prime}, y_{1}, y_{2}\right)\right\}=n^{-1} F\left(x^{\prime}, y_{1}, y_{2}\right), \\
\lambda\left\{u\left(n, x^{\prime}, Q\right)\right\}=n^{-1} G\left(x^{\prime}, Q\right) .
\end{gathered}
$$

Let $\bar{u}\left(n, n^{\prime}, y_{1}, y_{2}\right)$ be the sine transform of $u\left(n, x^{\prime}, y_{1}, y_{2}\right)$ with respect to $x^{\prime}$. When $n^{\prime}=n$, problem $\left(\mathrm{B}^{\prime}\right)$ becomes

$$
\begin{gathered}
-n^{2} \bar{u}\left(n, y_{1}, y_{2}\right)+\left[H_{0}\left(P_{0}\right)-(-1)^{n} H_{1}\left(P_{1}\right)\right] \\
+\Lambda\left\{\bar{u}\left(n, y_{1}, y_{2}\right)\right\}=n^{-1} f\left(n, y_{1}, y_{2}\right), \\
\lambda\{\bar{u}(n, Q)\}=n^{-1} g(n, Q) .
\end{gathered}
$$

If the equations in problem $\left(\mathrm{B}^{\prime \prime}\right)$ are multiplied by the parameter $n$, it becomes evident that problems $\left(\mathrm{A}^{\prime}\right)$ and $\left(\mathrm{B}^{\prime \prime}\right)$ are equivalent and that $n \bar{u}\left(n, y_{1}, y_{2}\right)$ is also a solution of $\left(\mathrm{A}^{\prime}\right)$. Suppose that the 
solution of $\left(\mathrm{A}^{\prime}\right)$ is unique, then

$$
v\left(n, y_{1}, y_{2}\right)=n \bar{u}\left(n, y_{1}, y_{2}\right) \text {. }
$$

Since $\bar{u}\left(n, y_{1}, y_{2}\right)$ is the iterated sine transform of $U\left(x, x^{\prime}, y_{1}, y_{2}\right)$, then according to our theorem of $\$ 2$,

$$
\begin{aligned}
u\left(n, y_{1}, y_{2}\right) & =S\left\{S\left\{U\left(x, x^{\prime}, y_{1}, y_{2}\right)\right\}\right\} \\
& =2^{-1} C\left\{U^{*}\left(x, y_{1}, y_{2}\right)\right\} .
\end{aligned}
$$

Hence in view of (8)

$$
\begin{aligned}
v\left(n, y_{1}, y_{2}\right) & =2^{-1} n C\left\{U^{*}\left(x, y_{1}, y_{2}\right)\right\} \\
& =-2^{-1} S\left\{\frac{\partial}{\partial x} U^{*}\left(x, y_{1}, y_{2}\right)\right\} .
\end{aligned}
$$

Since $v\left(n, y_{1}, y_{2}\right)=S\left\{V\left(x, y_{1}, y_{2}\right)\right\}$, then

$$
V\left(x, y_{1}, y_{2}\right)=-\frac{1}{2} \frac{\partial}{\partial x} U^{*}\left(x, y_{1}, y_{2}\right) .
$$

According to the definition (4) of the generalized convolution, this can be written as

$$
V\left(x, y_{1}, y_{2}\right)=\frac{1}{2} \frac{\partial}{\partial x} \int_{-\pi}^{\pi} U_{1}\left(x-x^{\prime}, x^{\prime}, y_{1}, y_{2}\right) d x^{\prime},
$$

where $U_{1}$ is an odd periodic extension of $U$ with respect to $x$ an odd extension with respect to $x^{\prime}$.

Formula (9) is then an extension of the Duhamel integral formula from time to space coordinates.

It can be shown that the above problem (B) can further be resolved into simpler problems, the solutions of which can be expressed in terms of some key functions. See [4].

\section{BIBLIOGRAPHY}

1. R. V. Churchill, Modern operational mathematics in engineering, McGraw-Hill, 1944.

2. Hans Kniess, Lösung von Randwertproblemen bei System gewöhnlicher Differentialgleichungen vermittels der endlichen Fourier-Transformation, Math. Zeit. vol. 44 (1938).

3. R. C. F. Bartels and R. V. Churchill, Resolution of boundary value problems by the use of a generalized convolution, Bull. Amer. Math. Soc. vol. 48 (1942) pp. 276-282.

4. A. W. Jacobson, A generalized convolution for the finite Fourier transformation, Thesis, University of Michigan, 1948.

UNIVERSITY OF MichigaN 